\title{
Les institutions du développement agricole durable des pays du Sud
}

\section{Michel Benoit-Cattin}

\section{(2) OpenEdition}

Journals

Édition électronique

URL : http://journals.openedition.org/economierurale/483

DOI : $10.4000 /$ economierurale.483

ISSN : 2105-2581

Éditeur

Société Française d'Économie Rurale (SFER)

Édition imprimée

Date de publication : 1 juin 2008

Pagination : 5-7

ISSN : 0013-0559

Référence électronique

Michel Benoit-Cattin, «Les institutions du développement agricole durable des pays du Sud », Économie rurale [En ligne], 303-304-305 | Janvier-juin 2008, mis en ligne le 30 juin 2010, consulté le 21 septembre 2020. URL : http://journals.openedition.org/economierurale/483 ; DOI : https://doi.org/ 10.4000/economierurale.483 


\title{
INTRODUCTION
}

\section{Les institutions \\ du développement agricole durable des pays du Sud}

\author{
Michel BENOIT-CATTIN • CIRAD, UMR Marchés, Organisations, Institutions et \\ Stratégies d'Acteurs (MOÏSA), Montpellier \\ Coordinateur du dossier
}

Co

es dernières années, l'économie du développement en général et celle de l'agriculture

en particulier ont été renouvelées en intégrant les préoccupations du développement durable et en prenant en compte les approches néoinstitutionnalistes.

Dans ce contexte, en novembre 2005, la SFER organisait à Montpellier des journées sur «Les institutions du développement durable des agricultures du Sud».

Les contributions reçues ont permis de faire un état des lieux des travaux de la recherche francophone sur le rôle des institutions dans le développement durable des agricultures du Sud.

Le terme «Institution » était entendu dans la tradition de l'économie institutionnelle, au sens de « règles du jeu ». Le champ géographique retenu était circonscrit aux pays du Sud, Maghreb et Proche-Orient compris. Après sélection, 52 communications ont été présentées et discutées pendant ces journées dont les actes ont été diffusés sous forme de Cédérom.

Selon les procédures éditoriales propres à l'édition de numéros thématiques issus des colloques organisés par la SFER, 12 contributions ont été expertisées et rassemblées dans ce numéro.

L'économie néoinstitutionnelle constitue un champ assez foisonnant et difficile à définir et délimiter. Elle trouve une application privilégiée dans des approches historiques et comparatives des processus de développement dans les pays du Sud.

Par rapport à un objet d'étude particulier, son environnement institutionnel pourra être pris en compte pour mieux comprendre sa propre dynamique. Il s'agit des normes et règles du jeu qui encadrent l'action individuelle et collective. L'action collective elle-même peut s'analyser en termes d'institutions, de règles que se donnent les acteurs, soit parce que l'environnement le permet, soit au contraire pour s'en protéger.

Ainsi dans l'article d'Omar Bessaoud sur «Les organisations rurales au Maghreb. Leur rôle dans le développement : un essai d'évaluation » d'une part et, d'autre part, celui de Jean-François Bélières en collaboration avec Michel Benoit-Cattin, Luc Barret, Hamady Djouara et Demba Kébé sur «Les organisations de producteurs en zone cotonnière au Mali. Conditions d'émergence et perspectives », les analyses des organisations de producteurs, respectivement en Afrique du Nord et au Mali, insistent sur l'importance du processus général de démocratisation à l'œuvre dans les sociétés concernées.

Dans le contexte thaïlandais, où la décentralisation est à l'ordre du jour, Cécile Barnaud en collaboration avec Guy Trébuil, Panomsak Promburom et François Bousquet, à propos de «La modélisation d'accompagnement pour une gestion concertée des ressources renouvelables en Thaïlande? » évaluent une méthode participative de gestion des ressources naturelles. 
En contrepoint, l'analyse de Jean-Michel Sourisseau, Philippe Bonnal et Perrine Burnod sur le «Changement institutionnel et agriculture à Mayotte. Les impacts d'une intégration renforcée à la République française sur l'activité agricole des ménages » montre qu'à Mayotte la transposition des règles de la République française ne suffit pas pour induire un processus de développement agricole similaire à celui observé en métropole.

À la suite de De Janvry et al. (1995) ${ }^{1}$, les analyses néoinstitutionnalistes du développement rural ont privilégié la prise en compte des rôles respectifs de l'État, du marché et des organisations civiles.

C'est dans cette optique que Marie-Hélène Dabat en collaboration avec Olivier Jenn-Treyer, Simon Razafimandimby et Louis Bockel, retracent dans leur article «L'histoire inachevée de la régulation du marché du riz à Madagascar » la difficile régulation du marché de cette denrée de base.

$\mathrm{Au}$ Mexique, c'est en se référant au nouveau rôle de l'État dans un contexte libéralisé que Virginie Brun explique dans son article « Secteur privé et céréaliculture familiale dans le Mexique du libre-échange. Une étude dans les terres basses du sud-Veracruz, le succès de la participation des petits producteurs au marché des céréales. Face à ce thème, au Sénégal, Papa Nouhine Dieye, en collaboration avec Etienne Montaigne, Guillaume Duteurtre et Jean-Pierre Boutonnet, insistent dans leur étude intitulée « Le rôle des arrangements contractuels dans le développement du système laitier local et des minilaiteries au Sénégal » sur le rôle que doit jouer l'État pour que les initiatives prises par les producteurs et petits transformateurs laitiers soient consolidées.

Si les articles cités insistent sur les vertus attendues ou constatées des changements institutionnels exogènes, d'autres interprètent les changements locaux comme des réactions de défense.

Ainsi, Elodie Maître D'Hôtel, Pierre-Marie Bosc et Johny Egg dans « L'action collective et la durabilité des filières agricoles au Costa Rica» montrent que les réactions face à la libéralisation des filières varient selon les filières et leur histoire. Pour le Mexique, Eric Léonard réinterprète, selon son analyse «Marché foncier, asymétries de pouvoir et exclusion. Incidences régionales des réformes libérales mexicaines à la fin du XIX siècle », l'histoire du foncier comme réaction, elle-même institutionnelle, à la libéralisation de la fin du XIX en mettant en évidence les inégalités induites.

Toujours pour ce qui est de la libéralisation du foncier, mais aujourd'hui en Côte d'Ivoire, Debegnoum Marcelline Soro et Jean-Philippe Colin analysent les nouveaux arrangements intrafamiliaux qui ménagent la transmission intergénérationnelle du patrimoine dans leur article «Marchandisation et individualisation des droits sur la terre en basse Côte d'Ivoire ».

Ces deux derniers textes, l'un en se préoccupant d'équité sociale, l'autre de transmission entre générations assurent une transition avec ceux privilégiant une entrée par le développement durable.

En effet, pour ce qui est du développement durable, à la suite du rapport Bruntland de 1987, on a d'abord insisté sur les responsabilités intergénérationnelles, réhabilitant les préoccupations de long terme dans le développement. À la suite de la Conférence de Rio de 1992, l'accent a davantage été mis sur les trois piliers d'égale importance : l'économique,

1. Alain De Janvry, Elisabeth Sadoulet, Eric Thorbecke (Eds.), State, Market, and Civil Organizations. London, Macmillan, 1995. 
le social et l'environnemental. Cependant, la référence au développement durable tend à privilégier ce troisième pilier au détriment des deux autres et de la dimension intergénérationnelle.

Les objectifs de développement durable se traduisent, notamment, par des normes venant enrichir l'environnement institutionnel des filières agricoles. Leurs effets peuvent être analysés en termes d'adaptation institutionnelle et évalués par rapport aux objectifs du développement durable. Le cas des filières bois et café au Brésil permet à MarieGabrielle Piketty, Maria-Célia Martins de Souza et Isabel Garcia Drigo, dans la "Certification environnementale et durabilité au Brésil: le café bio et le bois », de boucler cette boucle. C'est également ce que font Guy Faure, Sendy Veerabadren et Henri Hocdé dans leur article "L'agriculture familiale mise sous normes. Un défi pour les producteurs d'ananas au Costa Rica?».

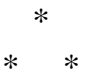

Dans l'ensemble, les processus de développement des agricultures du Sud sont significativement portés par les changements institutionnels, qu'ils soient exogènes ou endogènes, proactifs ou défensifs. Cependant, on ne saurait se contenter du seul constat que « les institutions, ça compte ». Une évaluation à l'aune du développement durable et plus particulièrement de ses trois piliers reste nécessaire tout en posant des problèmes de mesure qui pourraient faire l'objet de prochaines journées.

Que tous ceux qui ont contribué à ce numéro, les auteurs comme les referees soient ici remerciés chaleureusement de l'attention toute particulière qu'ils ont portée à ce thème. Nous laissons nos lecteurs puiser dans ces différentes recherches matière à leur propre réflexion. 